\title{
Longitudinal and transverse heating of a relativistic electron bunch induced by a storage ring free electron laser
}

\author{
M. Labat ${ }^{1}$ and M. E. Couprie ${ }^{1,2}$ \\ ${ }^{1}$ CEA, Service des Photons Atomes et Molécules, 91191 Gif-sur-Yvette, France \\ ${ }^{2}$ Synchrotron SOLEIL, Saint-Aubin, BP48, 91192 Gif-sur-Yvette CEDEX, France \\ M. Hosaka, A. Mochihashi, and M. Katoh \\ UVSOR Facility, Institute for Molecular Science, Okazaki 444-8585, Japan \\ Y. Takashima \\ Department of Material Processing Engineering, Graduate School of Engineering, Nagoya University, Chikusa-ku, \\ Nagoya 464-8603, Japan \\ (Received 23 May 2006; published 3 October 2006)
}

\begin{abstract}
The new trend is to operate storage ring based light sources in a "chromatic mode" with a distributed dispersive function in the straight sections for low emittance. The electron bunch heating induced by a storage ring free electron laser (FEL) has been investigated for such optics, and exhibits a more complex saturation process as compared to a usual achromatic mode of operation without dispersion in the straight sections. The correlated measured FEL power is then interpreted in terms of the electron bunch heating and compared to theoretical expectations. Experiments performed at UVSOR-II are here reported. The theoretical interpretation of the new saturation phenomenon is then discussed.
\end{abstract}

DOI: 10.1103/PhysRevSTAB.9.100701

PACS numbers: 41.60.Cr, 29.20.Dh

\section{INTRODUCTION}

Free electron lasers (FEL) are coherent tunable high brilliance light sources, based on the interaction of a relativistic electron bunch circulating in the permanent periodic magnetic field created by an undulator [1]. The spontaneous emission (synchrotron radiation emitted in the undulator) interacts with the electron beam and is amplified to the detriment of the kinetic energy. The light interplay induces an energy modulation inside the electron bunch, which is then transformed into a density modulation at the radiation wavelength, leading to a more coherent emission. It results in an emission of a polarized short pulse (femtosecond to picosecond) radiation. Tunability is achieved by changing the magnetic field of the undulator or the electron beam energy. FELs generally overcome in brilliance the performances of alternative light sources, such as synchrotron radiation [2], high harmonic in gases [3], x-ray laser based on collisionally pump x ray or on optical field ionized plasma amplifiers [4]. The oscillator configuration is the most common one for user applications in the infrared and in the ultraviolet spectral ranges. Presently, the record of the shortest wavelength for an FEL oscillator has been achieved on the European ELETTRA FEL, at $176 \mathrm{~nm}$ [5]. In parallel, a single pass FEL scheme such as self-amplified spontaneous emission [6] and high gain garmonic generation [7] is carried out in order to extend the spectral range to very short wavelengths. In the harmonic generation mode, $87 \mathrm{~nm}$ has been produced and exploited by users at BNL [8]. SASE radiation has been produced at the VUV-FEL (DESY) at
$32 \mathrm{~nm}$ [9] and the user program is launched. The relativistic electron bunch can be generated from a linear accelerator in which a fresh electron bunch interacts at each pass with the optical field of the laser light. Besides, on a storage ring free electron laser (SRFEL), the electron bunch is recirculating over many turns and keeps track of the interaction with the laser field from one pass to the other. In this case, the optical cavity length should be a submultiple of the revolution period, in order to ensure the synchronization between the electron bunches stored in the ring and the optical bunches bouncing in the optical cavity (the socalled "detuning condition"). The small signal gain is proportional to the electronic density, to the inverse of the cube of the electron beam energy $E$ and it depends on the insertion device length. The electronic density $n_{e}$ is related to the characteristics of the storage ring, and can be further increased by an appropriate choice of the electron beam optics. In particular, third generation light sources, in quest of very small emittances, tend to adopt optics with distributed dispersive function $\eta$ in the straight sections $[10,11]$. In addition to a renew of various components, the change towards chromatic optics allowed one to reduce the emittance $\varepsilon$ at UVSOR from 165 to 27.4 nmrad [12]. Generally, the available straight sections being rather short on a storage ring, one usually employs, instead of a simple undulator, an optical klystron (OK) [13]. It consists of two undulators of $N$ periods, separated by a dispersive section creating a wide wiggle of magnetic field. The energy modulation takes place in the first undulator, it is then transformed into bunching in the dispersive section, and coherent emission is produced in the second undulator. The 
spectrum results from the interference of the spectra of the two undulators, and the modulation rate (the equivalent to the optical contrast of the fringes) depends on the energy spread $\sigma_{\gamma}$ of the beam (i.e. the rms value of the electron beam distribution) [14]. The energy exchange between the FEL pulse and the electron beam leads to an enhancement of the electron beam energy spread, which is related to the FEL power, according to the so-called "Renieri limit" $[13,15,16]$. The enhancement of the energy spread is generally associated with an induced bunch lengthening in this case, and it has been observed on various storage ring FELs, such as ACO [17], VEPP3 [18], Super-ACO [19], ELETTRA [20], and UVSOR [21]. The FEL saturates via the increase of the energy spread and of the electron bunch length, leading to the gain reduction reaching the cavity losses value. The role played by the detuning in the saturation process has also been analyzed [22]. The FEL pulse being in fact shorter than the electron bunch length $\sigma_{l}$, the heating of the electron bunch is not uniformly distributed, and the electron refreshment occurs via the synchrotron motion [23,24]. Besides, the FEL-electron bunch interaction is usually accompanied by a competition between the FEL heating and the process leading to an increase of the electron bunch sizes, such as coherent synchrotron oscillations [25], microwave instability [26], sawtooth regimes [27], head-tail [28], and so on. The FEL interaction can even be considered as a stabilizing feedback on the electron beam itself. As a consequence, the FEL and storage ring should be seen more generally as a complete whole system. More recently, the influence of the FEL induced heating in terms of electron beam lifetime has been analyzed theoretically, for different regimes of operation [29] and partially measured in the Super-ACO case [30,31]. In particular, the case of a nondispersion free straight section for the FEL undulator has been considered. The energy spread heating is then associated with a local increase of the electron bunch transverse dimensions $\sigma_{x}$ and $\sigma_{z}$, leading to a further gain reduction towards saturation via the electronic density term, the Filling factor $F_{f}$ (representing the overlap between the transverse sizes of the electron bunch and the waist of the optical field). An extensive analysis of the electron heating phenomenon is here presented in the case of the UVSOR FEL operated in the visible at $600 \mathrm{MeV}$ (see characteristics in Table I), for which the electron beam optics can be easily adjusted for the chromatic and achromatic modes of operation. Dedicated electron beam measurements have been set up in order to properly characterize the FEL induced heating on the different electron beam features. The experimental results clearly indicate the new saturation process of the FEL, in the case of chromatic optics. A theoretical interpretation of the measured FEL power has also been carried out, confirms that the proposed saturation process involves both longitudinal and transverse dynamics, and provides further insight on the dynamical process. Such saturation
TABLE I. UVSOR-II machine operating point for FEL operation at $600 \mathrm{MeV}$, for the dispersion and dispersion free. $\beta_{x}$ and $\beta_{z}$ are the betatron functions, $Q$ the charge in the ring, $V^{\prime}$ the time derivative of the cavity voltage, $\nu_{s}$ the synchrotron tune, $\nu_{x}$, $\nu_{z}$ the betatron tunes, $\xi_{x}, \xi_{z}$ the natural chromaticities, $L$ the circumference of the ring, $N_{\mathrm{SP}}$ the number of super periods, $\alpha$ the momentum compaction factor, $\sigma_{l 0}$ the natural bunch length, $\sigma_{\gamma 0}$ the natural energy spread at quasizero current, and $\tau_{\mathrm{S}}$ the synchrotron damping time. For transverse sizes measurements, BL2A has an extraction angle of $4^{\circ}$ and BL3 $10^{\circ}$.

\begin{tabular}{|c|c|c|}
\hline & Achromatic & Chromatic \\
\hline$\varepsilon(\mathrm{nm} \mathrm{rad})$ & $40-38$ & 17.5 \\
\hline$\beta_{x}, \beta_{z}$ in $\mathrm{OK}(\mathrm{m})$ & $10 ; 2.8$ & $10 ; 3.1$ \\
\hline$\beta_{x}, \beta_{z}$ in BL2A (m) & $0.96 ; 9.03$ & $1 ; 9.46$ \\
\hline$\beta_{x}, \beta_{z}$ in $\operatorname{BL} 3(\mathrm{~m})$ & $0.89 ; 9.9$ & $0.53 ; 10.51$ \\
\hline$\eta$ in $\mathrm{OK}(\mathrm{m})$ & 0 & 0.8 \\
\hline$\eta$ in BL2A (m) & 0.54 & 0.26 \\
\hline$\eta$ in BL3 (m) & 0.54 & 0.19 \\
\hline$Q(\mathrm{C})$ & \multicolumn{2}{|c|}{$1.78 \times 10^{-30} I(\mathrm{~mA})$} \\
\hline$V^{\prime}\left(\mathrm{V} \mathrm{s}^{-1}\right)$ & \multicolumn{2}{|c|}{$3.11 \times 10^{13}$} \\
\hline$\nu_{s}$ & \multicolumn{2}{|l|}{$2.54 \times 10^{-3}$} \\
\hline$\nu_{x}, \nu_{z}$ & 3.68 & 3.23 \\
\hline$\xi_{x}, \xi_{z}$ & $-8.1 ;-7.3$ & $-6.1 ;-7.0$ \\
\hline$L(\mathrm{~m})$ & 53.2 & \\
\hline$N_{\mathrm{SP}}$ & 4 & \\
\hline$\alpha$ & 0.026 & 0.028 \\
\hline$\sigma_{\gamma 0}$ & 0.00034 & 0.00034 \\
\hline$\sigma_{l 0}(\mathrm{ps})$ & 101 & 105 \\
\hline$\sigma_{x}$ in $\mathrm{OK}(\mu \mathrm{m})$ & 616 & 500 \\
\hline$\sigma_{z}$ in $\mathrm{OK}(\mu \mathrm{m})$ & 103 & 70 \\
\hline$\tau_{\mathrm{S}}(\mathrm{ms})$ & 19 & \\
\hline
\end{tabular}

regimes are likely to be the general case for future FELs built on third generation synchrotron radiation storage rings, aiming at reaching very small emittances. Let us point out also the particular advantage of FELs with respect to other conventional lasers in having the possibility to completely characterize the amplifying medium. Indeed, the SRFEL dynamics is a complex phenomenon, going beyond the generation of coherent radiation for users.

\section{ELECTRON BEAM CHARACTERISTICS FOR FEL OPERATION AT UVSOR-II}

Storage ring free electron lasers were first implemented on first generation light sources such as ACO [32] (Orsay, France) where sextupoles just have been introduced in the magnetic optics, or on VEPP3 [33] (Novosibirsk, Russia) where a specific by-pass for the FEL insertion device has been constructed, and on TERAS [34] (Tsukuba, Japan). Then, SRFELs were installed either on dedicated racetrack shape rings such as DELTA [35], NIJI-IV [36], and DUKE [37], or on synchrotron radiation light sources of second generation, such as Super-ACO [38], UVSOR [39], and more recently of third generation such as ELETTRA [40]. The SRFELs on such synchrotron radiation facilities tend 
to quest for better compatibility with the current mode of operation. They also follow the evolution of LINAC based on and short wavelength FELs, in search of low emittance for high brilliance photon beam, leading also to an enhanced gain from a FEL point of view. On a storage ring, the natural emittance results from the balance between the radiation damping and the quantum fluctuations [41]. The double bend achromat (DBA) lattice is one of the most common structure for these machines [42]. The minimum of the emittance found under the achromatic conditions (the dispersion function and its derivative are zero at opposite ends of the dipole pair) can be further reduced by a factor of 3 by relaxing the condition of vanishing dispersion in the straight sections [43]. Such a strategy combining strong focusing, distributed dispersion function and chromaticities compensation has been adopted on Super-ACO [44] (Orsay, France), ESRF [45] (Grenoble, France), UVSOR-II [46] (Okazaki, Japan), and for new projects such as SOLEIL [47] (Saint-Aubin, France), DIAMOND [48] (Oxford, UK), Spring-8 [49] (Hyogo, Japan), AS [50] (Melbourne, Australia), and ALBA [51] (Barcelona, Spain). The FEL in such a case can benefit from a higher small-signal gain thanks to the reduced emittance and exhibit a new saturation process involving both longitudinal and transverse dynamics via the role played by the nonzero dispersion function in the optical klystron interaction region.

UVSOR accelerator complex consists of a $15 \mathrm{MeV}$ Linac, a $600 \mathrm{MeV}$ booster-synchrotron, and a $750 \mathrm{MeV}$ storage ring. UVSOR-II has a four fold symmetry DBA lattice, 8 straight sections $(4 \times 4 \mathrm{~m}$ and $4 \times 1.5 \mathrm{~m})$, and sextupoles integrated in the quadrupole magnets for making the straight sections as long as possible. UVSOR-II is equipped with a main cavity at $90.1 \mathrm{MHz}$ and a third harmonic cavity, which is detuned for FEL operation. We compare here the operation of the SRFEL on UVSOR-II using chromatic and achromatic lattice at $600 \mathrm{MeV}$. In the FEL mode of operation, two bunches are stored. Table I presents the characteristics of the UVSOR-II electron beam in both cases. Distributing the dispersion function leads to an emittance reduced by a factor of 2 , without changing the betatron tunes. Figure 1 shows the optical functions of UVSOR-II for both cases, with the betatron function and the dispersion function distributed in the straight sections.

The transverse sizes of the beam have been measured versus the stored current with an imaging technique at two different bending magnets ports in the ring: BL2A and BL3. The optical functions are presented in Table I. Measurements performed at $750 \mathrm{MeV}$ lead to a horizontal beam size of $250 \mu \mathrm{m}$, in agreement with the prediction from SAD (strategic accelerator design code [52] developed at KEK [53]). Figure 2 presents the transverse sizes measurements performed at $600 \mathrm{MeV}$ for the two operating points. The transverse sizes of the electron beam, at the

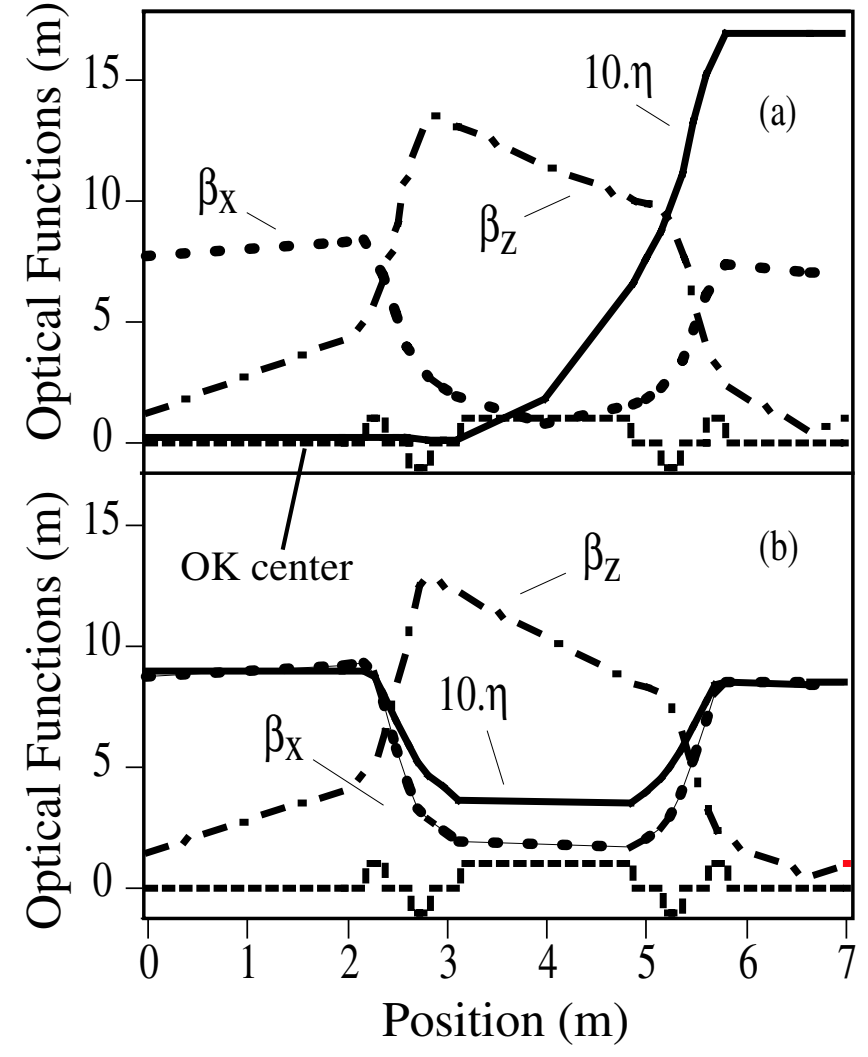

FIG. 1. Optical functions of UVSOR-II at $600 \mathrm{MeV}$ : (a) achromatic optics, (b) chromatic optics. Dashed line: position of the magnets.

longitudinal position $s$ in the ring, are related to the emittance $\varepsilon$, the magnetic dispersion $\eta$, and betatron function $\beta$ by:

$$
\left\{\begin{array}{l}
\sigma_{x}(s)=\sqrt{\varepsilon_{x}(s) \beta_{x}(s)+\eta_{x}^{2}(s) \sigma_{\gamma}^{2}} \\
\sigma_{z}(s)=\sqrt{\varepsilon_{z}(s) \beta_{z}(s)} .
\end{array}\right.
$$

The horizontal beam size is smaller in the chromatic case, because of the reduction of emittance and the very small value of the dispersion in BL3. When the stored beam current increases, the transverse sizes remain constant until a given threshold where transverse instabilities start to be dominant. Such instabilities can be damped with an appropriate setting of the chromaticities.

The bunch length $\sigma_{l}$ has been measured with a double sweep streak camera versus current [see Fig. 3(a)]. A bunch lengthening is observed, as the current increases. No difference in bunch length is observed for the chromatic and achromatic cases. Figure 3(b) shows the deformation of the bunch shape for different values of the current. As the current increases, the bunch gets larger. At $38 \mathrm{~mA}$, the shape is symmetric whereas at $80 \mathrm{~mA}$ the leading edge of the distribution is sharper than the falling edge. The energy spread $\sigma_{\gamma}$ can be accessed by two different methods. The first one consists in measuring the optical klystron spec- 


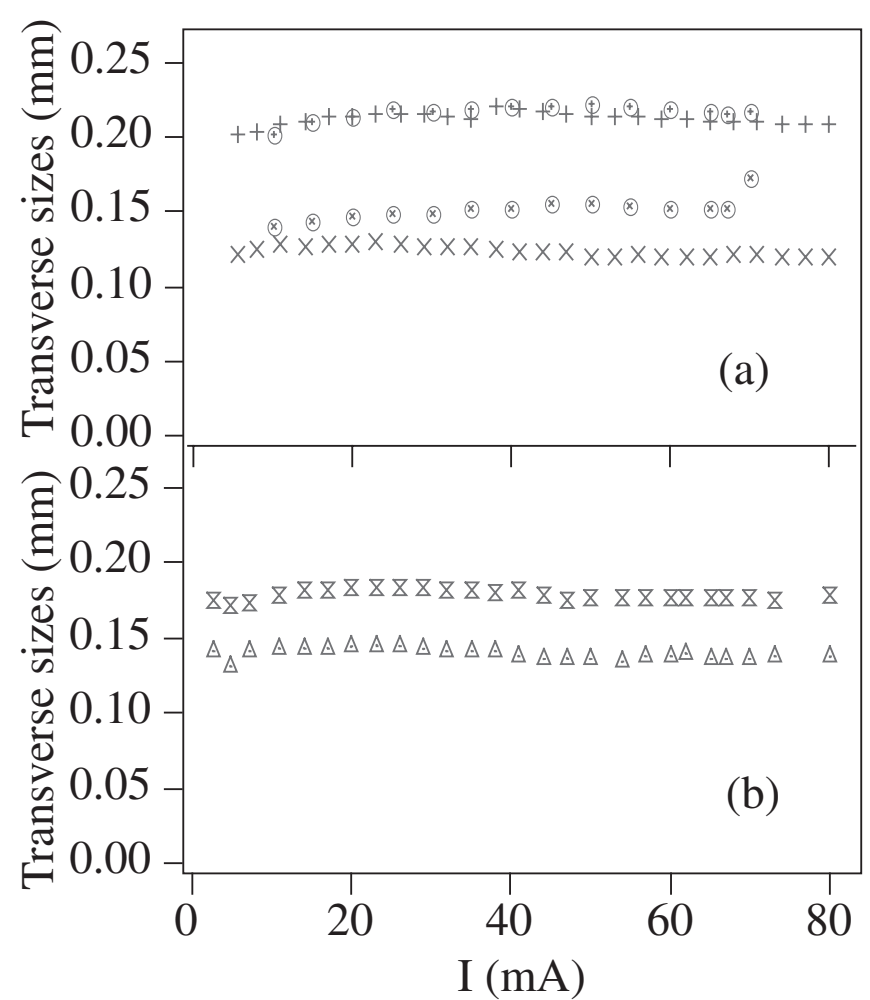

FIG. 2. Transverse size measurements performed at BL3: (a) achromatic optics (two series of measurements): $(+)$ and $(\oplus)$ for $\sigma_{x},(\times)$ and $(\otimes)$ for $\sigma_{z},\left(\right.$ b) chromatic optics: $\sigma_{x}:(\nabla)$ and $\sigma_{z}$ : $(\triangle)$. Data have been treated taking into account the linearity of the employed CCD camera (Vixen C004-3M). Measurement accuracy: $3 \%$.

trum, which exhibits interferences between the radiation emitted by each undulator section. The modulation rate (i.e. the contrast of the fringes) is directly related to the energy spread, as in the following:

$$
f=f_{0} \exp \left[-8 \pi^{2}\left(N+N_{d}\right)^{2} \sigma_{\gamma}^{2}\right]
$$

with $f_{0}$ the residual modulation rate due to the imperfection of the magnetic field, the transverse sizes and divergence of the electron beam, and the resolution of the monochromator, $N+N_{d}$ is the interference order due to the dispersive section. For these experiments, the undulators have been set up to the helical mode to limit the mirror degradation with only the first harmonic emitted on axis. The characteristics of the UVSOR-II optical klystron for FEL operation are given in Table II. Besides, the energy spread can be deduced from the transverse size measurements [see Eq. (1)] in a location where the dispersion function is different from zero, as in the case of BL3 for the achromatic and chromatic cases previously mentioned. Figure 4 illustrates the energy spread versus current measured with the first method. In the case of the chromatic operation point, the natural (i.e. near zero beam current) energy spread and the bunch length measurements are in agreement with theoretical expectations, according $\sigma_{l 0}=$

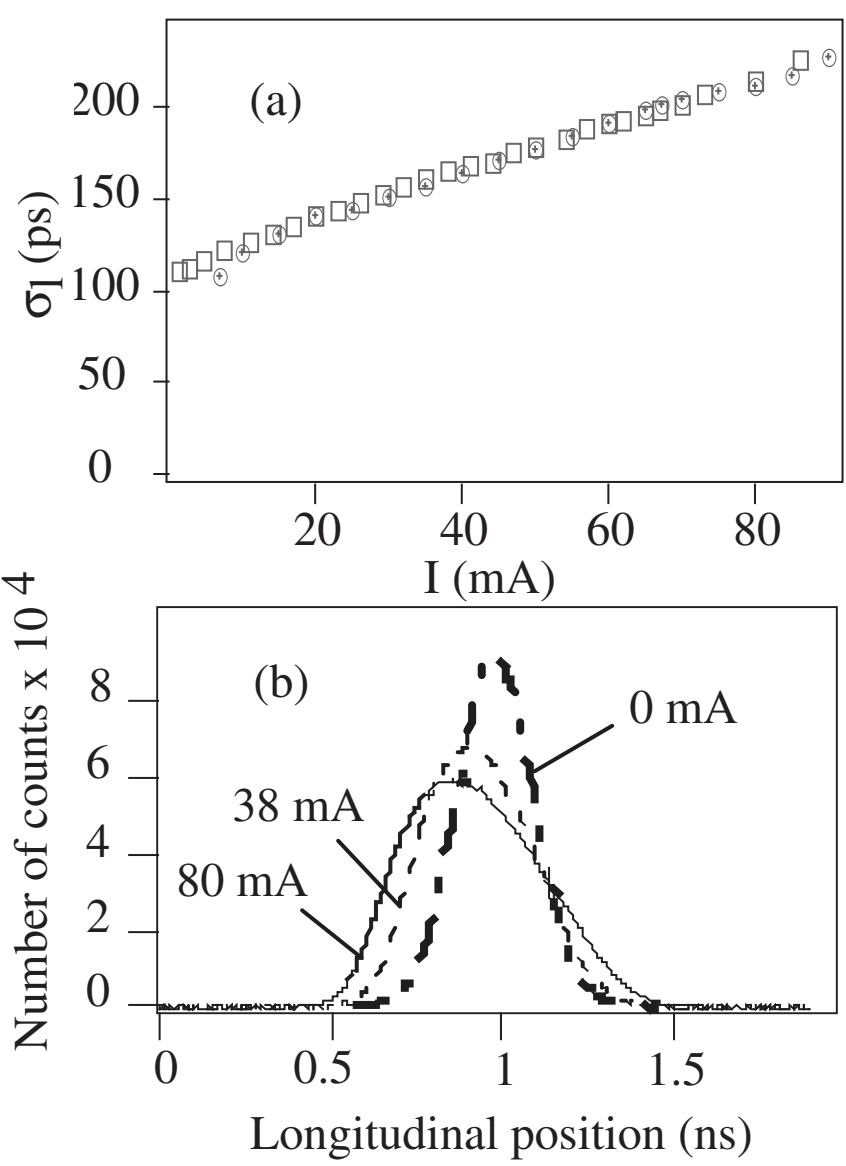

FIG. 3. (a) The rms bunch length versus current, in the achromatic $(\oplus)$ and chromatic $(\square)$ cases with FEL off, measured with a double sweep streak camera Hamamatsu C5680. (b) Evolution of the electron beam longitudinal profile for different currents (chromatic case) with FEL off (data analysis from streak camera Hamamatsu C5680 images). Continuous line: $I=80 \mathrm{~mA}$, dashed line: $I=38 \mathrm{~mA}$, dotted and dashed line: $I=0 \mathrm{~mA}$.

$\alpha c\left(\sigma_{\gamma 0} / w_{S}\right)$, where $w_{S}=\sqrt{\left(e \alpha V_{\mathrm{RF}} w_{\mathrm{RF}}\right) /\left(T_{0} E\right)}$ is the synchrotron angular frequency, $T_{0}$ is the revolution period, $e$ the electronic charge, $E$ its energy, $w_{\mathrm{RF}}$ its angular frequency, $c$ the speed of light, and $V_{\mathrm{RF}}$ the radio frequency (RF) cavity voltage. Collective effects appear when the beam current is increased, under the influence of the electron beam induced wakefield reflecting in the vacuum

TABLE II. UVSOR-II optical klystron characteristics. $\lambda_{0}$ is the period of the undulator, $K$ the undulator parameter, $L_{\text {disp }}$ the dispersive section length, and $L_{\mathrm{OK}}$ the total length.

\begin{tabular}{ll}
\hline \hline$N$ & 9 \\
Gap (mm) & $30-150$ \\
Remanent field (T) & 1.3 \\
$K$ (helical) & $0.07-4.6$ \\
$K$ (linear) & $0.15-8.5$ \\
$L_{\text {disp }}(\mathrm{mm})$ & 302.5 \\
$L_{\mathrm{OK}}(\mathrm{mm})$ & 2351.2 \\
\hline \hline
\end{tabular}



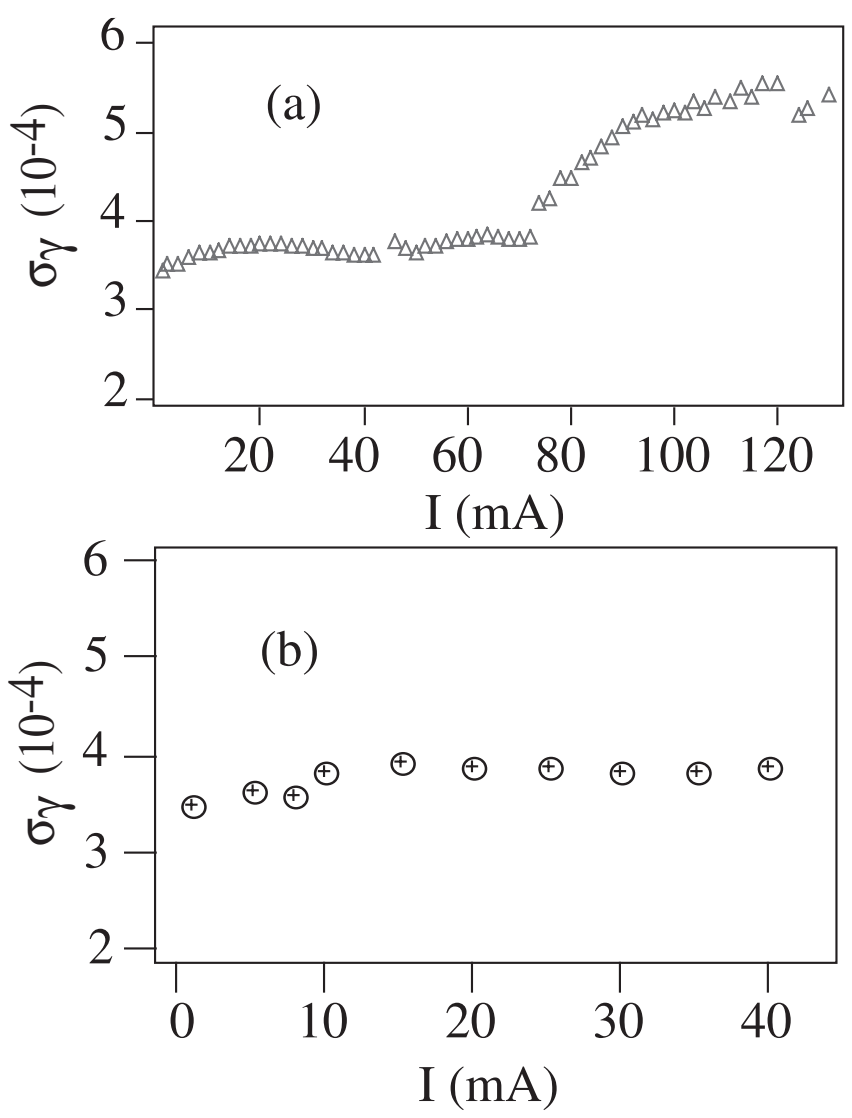

FIG. 4. Energy spread versus current from the optical klystron method: (a) in the chromatic case (for $I=0$ to $120 \mathrm{~mA}$ ), (b) in the achromatic case (for $I=0$ to $40 \mathrm{~mA}$ ).

chamber and perturbating back the electron bunch. In the low current region, an equilibrium situation can be reached with a proper balance between the excitation and damping of the perturbation, leading to an increase of the equilibrium bunch dimensions. The nonsymmetrical shape of the electron bunch distribution seen in Fig. 3(b) is due to its deformation induced by the wakefield. Up to $70 \mathrm{~mA}$ per bunch, the energy spread remains equal to its zero current theoretical value of $0.035 \%$, whereas the bunch length is continuously increasing with current. In this current range, the electron beam longitudinal dynamics is dominated by potential well distortion [54], due to the distortion of the RF cavity field by the electron bunch wakefield. It results in a lengthening of the electron bunch [55] but the energy spread remains unmodified. Above a threshold of $70 \mathrm{~mA}$ per bunch, both energy spread and bunch length increase with current and the dynamics comes into a regime of microwave instability, resulting from the electron beam interaction for which the wavelength of the wakefield lies in the microwave region. In this regime, a clear deformation of the electron beam distribution can be seen. The threshold of the wakefield is given by the Boussard criterium [56]: $\sigma^{2}<\sigma_{\text {th }}^{2}=\left(k_{I E} / k_{L I}\right)^{4 / 3}-1$, where $k_{I E}=$ $\frac{w_{c}}{2 \pi} \sqrt{\left[(2 \pi)^{1 / 2} I_{0}\left|\frac{Z_{n}}{n}\right|\right] /\left(\frac{E}{e} \sigma_{\gamma 0}\right)}$ is the instability excitation pa- rameter, with $w_{c}$ the bunch cut off frequency, $I_{0}$ the average current, and $\left|\frac{Z_{n}}{n}\right|$ the ring normalized longitudinal impedance. $k_{L I}$ is the Landau instability damping parameter, described by $k_{L I}=w_{c} \alpha \sigma_{\gamma 0}$. From Boussard criterium, a critical impedance $\left|\frac{Z_{n}}{n}\right|_{\text {crit }}=1.2 \Omega$, and an effective impedance $\left|\frac{Z_{n}}{n}\right|_{\text {eff }}=4.2 \Omega$ [57] can be deduced.

A slight transverse instability may also appear. The achromatic operation shows a similar behavior, with no significant change in the bunch length behavior versus current. Above $35 \mathrm{~mA}$ per bunch, a strong vertical headtail instability appeared and the threshold of microwave instability could not be characterized.

\section{GAIN EVOLUTION AT UVSOR-II}

The gain is defined as the relative increase of the laser power for one passage of the electron beam in the interaction region, i.e. in the optical klystron. The FEL grows from the small signal gain, which is maximum for start-up. The initial gain is determined by the electron beam characteristics versus current, as described in the previous section, and by the optical klystron characteristics. At saturation, the gain reaches the level of the cavity losses after being reduced by the heating of the electron beam induced by the FEL. Storage ring free electron lasers are usually operating under small-gain regimes, because of the limited length of the available straight section for the undulators. The small signal gain can then be expressed by the Madey's theorem [58] as the derivative of the spontaneous emission versus the energy. The gain at the frequency $\omega$, in the helical optical klystron case, can be expressed as:

$$
G=\frac{r_{e} 4 \pi^{5 / 4} N_{e}}{\sigma_{x} \sigma_{z} \sigma_{l}}\left(K_{x}{ }^{2}+K_{z}{ }^{2}\right) N^{3} \lambda_{0}{ }^{2} \frac{F_{f}}{\gamma^{3}} \frac{\partial}{\partial \delta_{1}}\left[\sin c\left(\delta_{1}\right)^{2}\right]
$$

with $r_{e}$ the classical radius of the electron, $N_{e}$ the number of electrons per bunch, $K_{x, z}$ the undulator parameters, $\delta_{1}=$ $\pi N\left(1-\frac{\omega}{\omega_{R}}\right)$ and $\omega_{R}$ the resonance frequency of the FEL. In addition, the Filling factor $F_{f}$ [59] term represents the overlap between the electron beam and the optical wave of the light of waist $w_{0 x}$ and $w_{0 z}$, for ensuring the proper transverse interaction. It is usually expressed as:

$$
F_{f}=\frac{1}{\sqrt{\left[1+\left(\frac{w_{0 x}}{2 \sigma_{x}}\right)^{2}\right]\left[1+\left(\frac{w_{0 z}}{2 \sigma_{z}}\right)^{2}\right]}} .
$$

A correction term $f_{C}\left(w_{0}, \sigma_{x}, \sigma_{z}\right)$ depending on the laser and electron beam dimensions has been added [60], in order to consider the horizontal wiggling trajectory of the electron beam in the undulator and a Gaussian light beam. From the simulations, an empirical analytical expression has been deduced as [61]: 
TABLE III. Values of the empirical parameters of the Filling factor expression.

\begin{tabular}{lcccc}
\hline \hline$\sum$ & $a$ & $b$ & $c$ & Correlation coefficient \\
\hline 0.071 & 3.0197 & 1.3030 & 0.1560 & 0.99779 \\
0.14 & 3.0062 & 1.2720 & 0.1532 & 0.99844 \\
0.24 & 2.6920 & 1.1230 & 0.1524 & 0.99866 \\
0.35 & 2.6358 & 0.9472 & 0.1528 & 0.99825 \\
0.5 & 2.1689 & 0.5900 & 0.1845 & 0.99956 \\
0.71 & 2.0402 & 0.5064 & 0.1930 & 0.99354 \\
1.41 & 1.6681 & 0.2578 & 0.3983 & 0.92294 \\
2.35 & 1.5264 & 0.1757 & 0.9481 & 0.91290 \\
\hline \hline
\end{tabular}

$$
F_{f}=\prod_{i=x}^{z} \frac{\sqrt{a W_{i}}}{\left(1+b W_{i}^{2}\right) \sqrt{\left(1+\frac{c}{\Sigma_{i}^{2}}\right)}}
$$

with $\quad W_{X}=w_{0 x} \sqrt{\frac{\pi}{\lambda L_{k o}}}$ (respectively $W_{Z}$ ) and $\Sigma_{X}=$ $\sigma_{x} \sqrt{\pi / \lambda L_{k o}}$ (respectively $\Sigma_{Z}$ ), $Z_{o}$ is the Rayleigh range, $\lambda$ the laser wavelength, $L_{\mathrm{OK}}$ the length of the optical klystron, and $a, b, c$ the constants deduced from a least square numerical fit given in Table III.

The FEL gain deduced from the electron beam measurements is illustrated in Fig. 5. In this case, the rms value of the bunch length has been used for the estimation of the electronic density, the calculated transverse sizes of the electron beam in the undulator, and the energy spread deduced from the optical klystron spectra analysis.

A gain increase with the current can be observed, and two types of evolution can be distinguished. From 0 to $70 \mathrm{~mA}$, the electronic density proportional to $\frac{N_{e}}{\sigma_{x} \sigma_{z} \sigma_{l}}$ increases with the current, while the energy spread, as mentioned previously, remains constant. The gain grows nearly as a proportional function of the beam current. Above $70 \mathrm{~mA}$, the enhancement of the energy spread leads to a

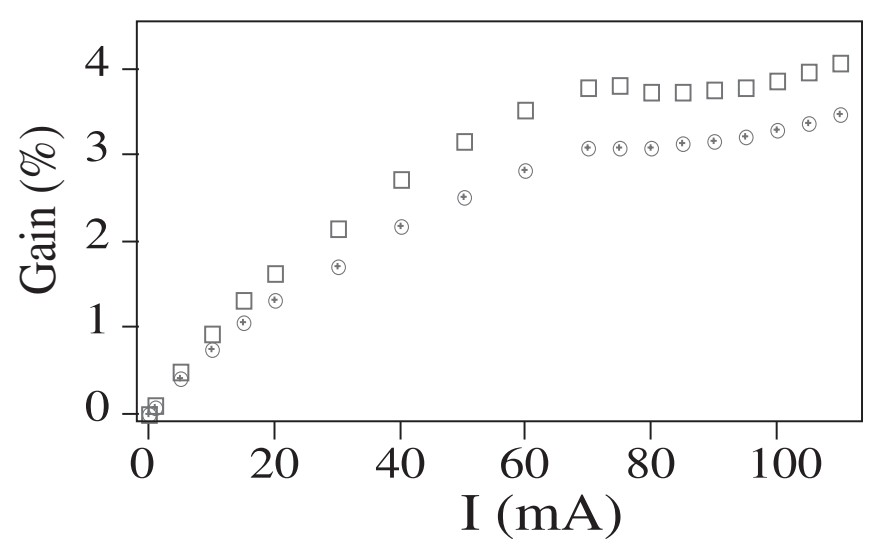

FIG. 5. (Color) Gain versus current in the achromatic $(\oplus)$ and chromatic ( $\square$ ) cases. Assumed coupling between the $x$ and $z$ dimensions: $10 \%\left(\sigma_{x}=616 \mu \mathrm{m}, \sigma_{z}=103 \mu \mathrm{m}\right.$ in the achromatic case, and $\sigma_{x}=500 \mu \mathrm{m}, \sigma_{z}=70 \mu \mathrm{m}$ in the chromatic case), deduced from experimental measurements. relative gain reduction via the modulation rate term, balancing the increase of peak current.

The gain calculated using the electron beam measurements can be confirmed by the laser threshold current values measured for different cavity losses $P$. The measured threshold current are for the achromatic point $5.8 \mathrm{~mA}$ (respectively $4.7 \mathrm{~mA}, 1.8 \mathrm{~mA}$ ) for $P=0.27 \%$ (respectively $0.12 \%, 0.11 \%$ ), and for the chromatic point $6 \mathrm{~mA}$ for $P=0.32 \%$.

As expected with the quest of low emittances for FEL, the gain comparison for the two optics shows here a larger gain in the chromatic case, related to the reduction from the betatron contribution to the transverse sizes in the FEL straight section, even though it is slightly counterbalanced by the increase of the dispersion contribution in the undulator. The ratio of the gain in the chromatic and achromatic case increases with current when the potential well distortion is dominant, whereas the ratio is constant in the microwave instability regime.

\section{ELECTRON BEAM HEATING INDUCED BY THE FEL}

The energy exchange between the electron beam and the FEL optical field leads to a local heating of the electrons interacting with the short pulse light, which is transferred to the whole bunch via synchrotron oscillation. As a result, the overall rms energy spread of the beam increases and the gain is consequently reduced and reaches the values of the cavity losses at equilibrium. Figure 6 shows the horizontal beam sizes in presence of the FEL. In the achromatic case, the transverse bunch sizes remain unmodified. In the chromatic case, because of the presence of a nonzero dispersion function in the optical klystron straight section, the horizontal beam size also increases, and the vertical bunch size gets larger via the coupling between the two transverse dimensions. The saturation in this case now involves modifications of the electron beam features in the transverse plane in the FEL straight section, and not only in the longitudinal one (enhancement of energy spread and associated bunch lengthening) as in the achromatic case. It results in a further decrease of the gain via the electronic density and the Filling factor terms, when the laser intensity gets larger. As a consequence, the required amount of increase of energy spread to get the FEL saturated is smaller than in the achromatic case.

Figure 7(a) shows the increase of energy spread in the achromatic optics case versus current. According to Eq. (3) and Fig. 5, a higher current implies a larger initial gain. A more important reduction of the gain is then required to reach the losses level, and as a consequence a larger energy spread enhancement takes place. Figure 7 also compares two cases of cavity losses corresponding to different experimental sessions. The higher the losses, the higher is the gain at saturation, and the smaller is the enhancement of the energy spread. The electron and photon beams inter- 


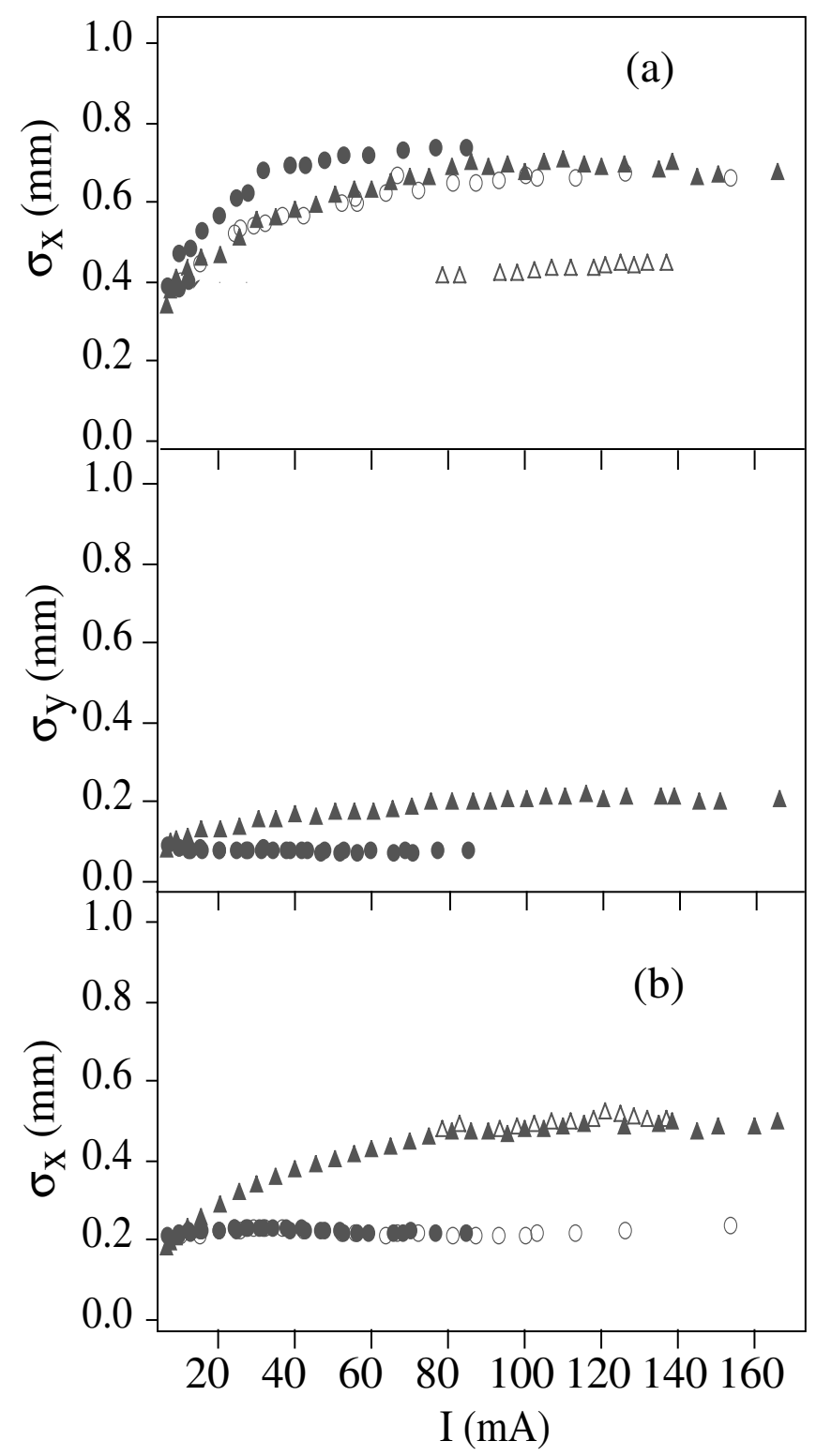

FIG. 6. Transverse size versus current FEL on, measured on (a) BL2 and (b) BL3, under different conditions: achromatic optics: (O) $P=0.3 \%,(\bigcirc) P=0.12 \%$; chromatic optics: $(\mathbf{\Delta}) P=$ $0.3 \%,(\triangle) P=0.12 \%$.

action being less intense, the resulting energy spread is smaller. For the chromatic operation, the energy spread can be deduced from the measurements of $\sigma_{x}$, while considering the increase of emittance deduced from the $\sigma_{y}$ behavior. In the chromatic case, Fig. 7(b) shows that the FEL induced energy spread is smaller. The correlated bunch lengthening is also reduced (see Fig. 8), for the same values of the cavity losses. The enhancement of energy spread is correlated to a bunch lengthening, as illustrated in Fig. 8. The change of bunch length also contributes to the gain reduction via the electronic density term. Again, the smaller the cavity losses, the smaller the gain and so the more the electron bunch is heated, as shown in Fig. 8.

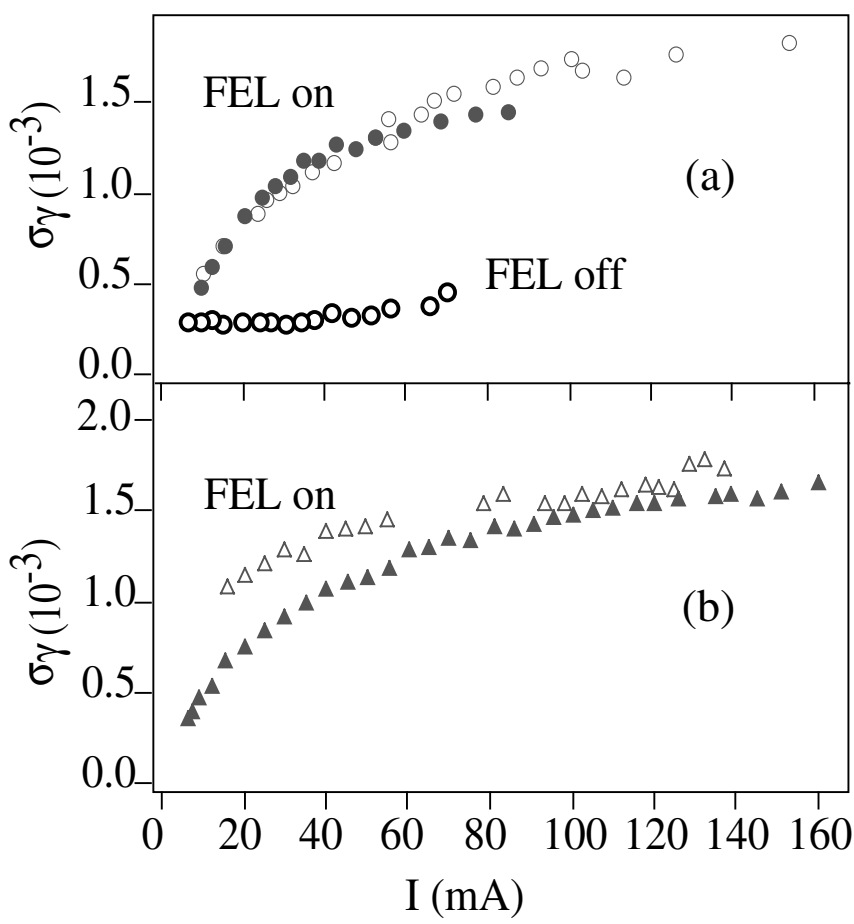

FIG. 7. Energy spread in the presence of FEL for different losses values, deduced from the transverse size measurements. (a) Achromatic optics: (O) $P=0.3 \%$, (○) $P=0.12 \%$. (b) Chromatic optics case: $(\boldsymbol{\Delta}) P=0.3 \%,(\triangle) P=0.12 \%$.

This interpretation is further confirmed by the modeling of the FEL dynamics using the code LAS developed in Orsay $[62,63]$ under MATLAB software. Different codes describing the dynamics of storage ring FEL have been developed [64]. The LAS code follows the pass to pass evolution of the laser intensity and of the electron beam characteristics. It has been recently modified to take into account the influence of the nonzero dispersion function in

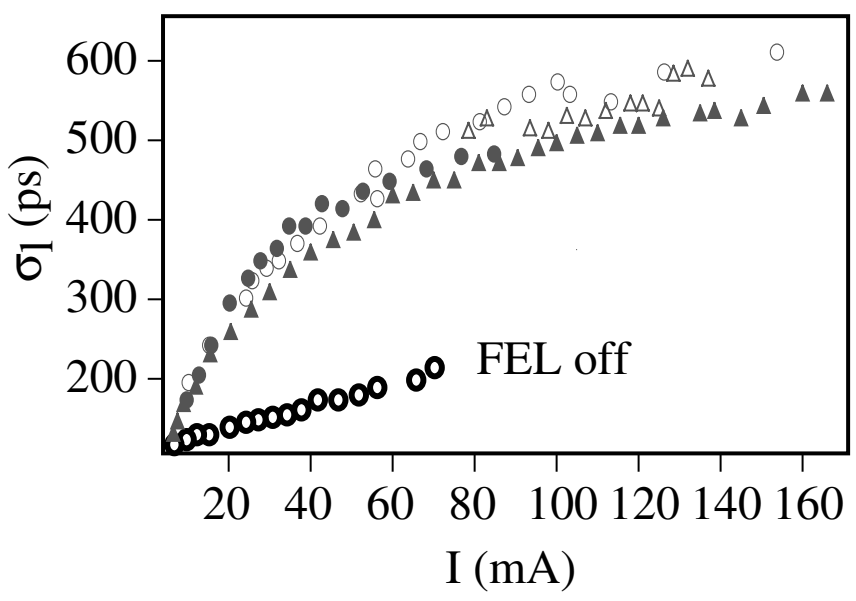

FIG. 8. Bunch length (rms) versus current with FEL on. Achromatic optics: $(\bigcirc) P=0.3 \%,(\bigcirc) P=0.12 \%$. Chromatic optics: $(\mathbf{\Delta}) P=0.3 \%,(\triangle) P=0.12 \%$. 
the optical klystron interaction region. The FEL longitudinal dynamics is described by three fundamental equations enabling the calculation of the laser intensity and gain, and of the energy spread. The laser intensity profile $y_{n}(\tau)$ is given at $n$th pass by the following heuristic equation:

$$
y_{n}(\tau)=(1-P) y_{n-1}(\tau)\left[1+g_{n}(\tau)\right]+i_{s n}(\tau),
$$

where $\tau$ is the temporal position with respect to the center of the electronic distribution. The first term represents the light amplification by a gain $g_{n}(\tau)$ from the previous pass reflected on both mirrors of the optical cavity. In addition, at each pass is added the spontaneous emission $i_{s n}$ produced at the $n$th pass in the optical klystron, and amplified by the gain $g_{n}(\tau)$ of the $n$th electron-photon interaction. The gain $g_{n}(\tau)$ at pass $n$ for a longitudinal position $\tau$ and a detuning $\delta$ is given by:

$$
\begin{aligned}
g_{n}(\tau)= & g_{i} \frac{\sigma_{x 0} \sigma_{z 0} \sigma_{l 0}}{\sigma_{x n} \sigma_{z n} \sigma_{\ln }} \frac{F_{f n}}{F_{f 0}} \exp \left[-k\left(\sigma_{\gamma n}^{2}-\sigma_{\gamma 0}^{2}\right)\right] \\
& \times \exp \left(-\frac{(\tau+\delta)^{2}}{2 \sigma_{\ln }^{2}}\right) .
\end{aligned}
$$

$g_{i}$ is the maximum gain when the laser is off for perfect tuning, obtained with Eq. (3). The second term involving the transverse and longitudinal beam sizes for initial conditions (index 0) and at pass $n$ (index n) represents the electronic density contribution to the gain. The third term, the filling factor, represents the transverse overlap between the electron beam and the light pulse for initial condition and at pass $n$. The next contribution comes from the modulation term of the optical klystron [see Eq. (2)] and plays a major role in the FEL saturation, since the gain due to the heated energy spread is exponentially reduced. The constant $k$ can be expressed in terms of interference order using Eq. (2). Finally, the last term represents the detuning gain profile reproducing the electron beam distribution, which is taken to be Gaussian in a first approximation. In this model, the slippage (defined as the delay between the electron bunch and the light pulse due to their velocity difference, at the end of the optical klystron) is being neglected since it is extremely small.

At equilibrium (index $e$ ), for perfect tuning $(\delta=0)$, the gain reaches the cavity losses value $P$ :

$$
P=g_{i} \frac{\sigma_{x 0} \sigma_{z 0} \sigma_{l 0}}{\sigma_{x e} \sigma_{z e} \sigma_{l e}} \frac{F_{f e}}{F_{f 0}} \exp \left[-k\left(\sigma_{\gamma e}^{2}-\sigma_{\gamma 0}^{2}\right)\right] .
$$

In addition, the relation of proportionality: $\sigma_{l}=\alpha c \frac{\sigma_{\gamma}}{w_{S}}$, is assumed to be valid whatever the electron beam current, so the bunch length can be expressed in function of the energy spread, and $\sigma_{l 0}, \sigma_{l e}$, and $\sigma_{\ln }$ be replaced by $\sigma_{\gamma 0}, \sigma_{\gamma e}$, and $\sigma_{\gamma n}$. This new version of LAS introduces a direct calculation of the energy spread at equilibrium with Eq. (8), whereas an estimated value of the energy spread at saturation was introduced before.
The evolution of the normalized energy spread $\Sigma_{n}=$ $\left(\sigma_{\gamma n}^{2}-\sigma_{\gamma 0}^{2}\right) /\left(\sigma_{\gamma e}^{2}-\sigma_{\gamma 0}^{2}\right)$ is given by:

$$
\Sigma_{n}=\Sigma_{n-1}+\frac{2 T_{0}}{\tau_{s}}\left(I_{n-1}-\Sigma_{n-1}\right)
$$

where $I_{n-1}$ is the normalized intensity stored in the cavity at $n-1$ th pass, defined as $I_{n-1}=\int_{-\infty}^{+\infty} y_{n-1}(\tau) d \tau$. The term $\frac{2 T_{0}}{\tau_{s}} I_{n-1}$ reflects the heating of the electron bunch by the laser intensity, whereas $-\frac{2 T_{0}}{\tau_{s}} \Sigma_{n-1}$ reflects the damping of the synchrotron oscillations with the synchrotron damping time $\tau_{S}$ and the electron bunch revolution period $T_{0}$.

By replacing the equilibrium gain $g_{e}$ equal to the cavity losses $P$ in Eq. (7), and using the expression of the normalized energy spread, a more implicit equation for the gain at pass $n$ is obtained:

$$
\begin{aligned}
g_{n}(\tau)= & g_{i} \frac{\sigma_{x 0} \sigma_{z 0} \sigma_{\gamma 0}}{\sigma_{x n} \sigma_{z n} \sigma_{\gamma n}} \frac{F_{f n}}{F_{f 0}}\left(\frac{P}{g_{i}}\right)^{\Sigma_{n}}\left(\frac{\sigma_{x e} \sigma_{z e} \sigma_{\gamma e}}{\sigma_{x 0} \sigma_{z 0} \sigma_{\gamma 0}} \frac{F_{f 0}}{F_{f e}}\right)^{\Sigma_{n}} \\
& \times \exp \left(-\frac{(\tau+\delta)^{2}}{2 \sigma_{\tau}^{2}}\right) .
\end{aligned}
$$

The FEL dynamical behavior is simulated with the modified version of LAS, now taking into account the transverse dimensions of the electron beam and the proper energy spread heating leading to the FEL saturation, based on Eqs. (6), (9), and (10).

Figure 9(a) shows the evolution of the relative energy spread calculated with LAS versus the passage number for perfect tuning. After damped oscillations of relaxation, the energy spread reaches its equilibrium value. For $0.3 \%$ cavity losses, the equilibrium energy spread in the chromatic case reaches $1.5 \sigma_{\gamma 0}$, whereas it reaches $1.6 \sigma_{\gamma 0}$ in the achromatic case. Such a difference is in qualitative agreement with the experimental observation at $0.3 \%$ losses: in the chromatic case $1.5 \sigma_{\gamma 0}$ and in the achromatic case $1.65 \sigma_{\gamma 0}$. Figure 9 also illustrates that for both magnetic configurations, higher cavity losses value lead to smaller induced energy spread since lower gain is required for saturation, in agreement with the experimental results of Fig. 7.

SRFELs have a pulsed structure at a high repetition rate, resulting from the passes of the stored bunches in the storage ring. But depending on the detuning, it can also produce series of macropulses at the ms range. As the detuning increases, the laser goes through a continuous mode of emission, followed by a pulsed one, another continuous one, before the intensity falls to zero. This evolution is simulated in Fig. 9, where the successive regimes are presented. The simulation also reveals that in the chromatic case, the width of the pulsed mode is smaller, due to the reduction of the bunch length [see Eq. (6)]. This phenomenon will be investigated into details in a further work. 


\section{Time (ms)}

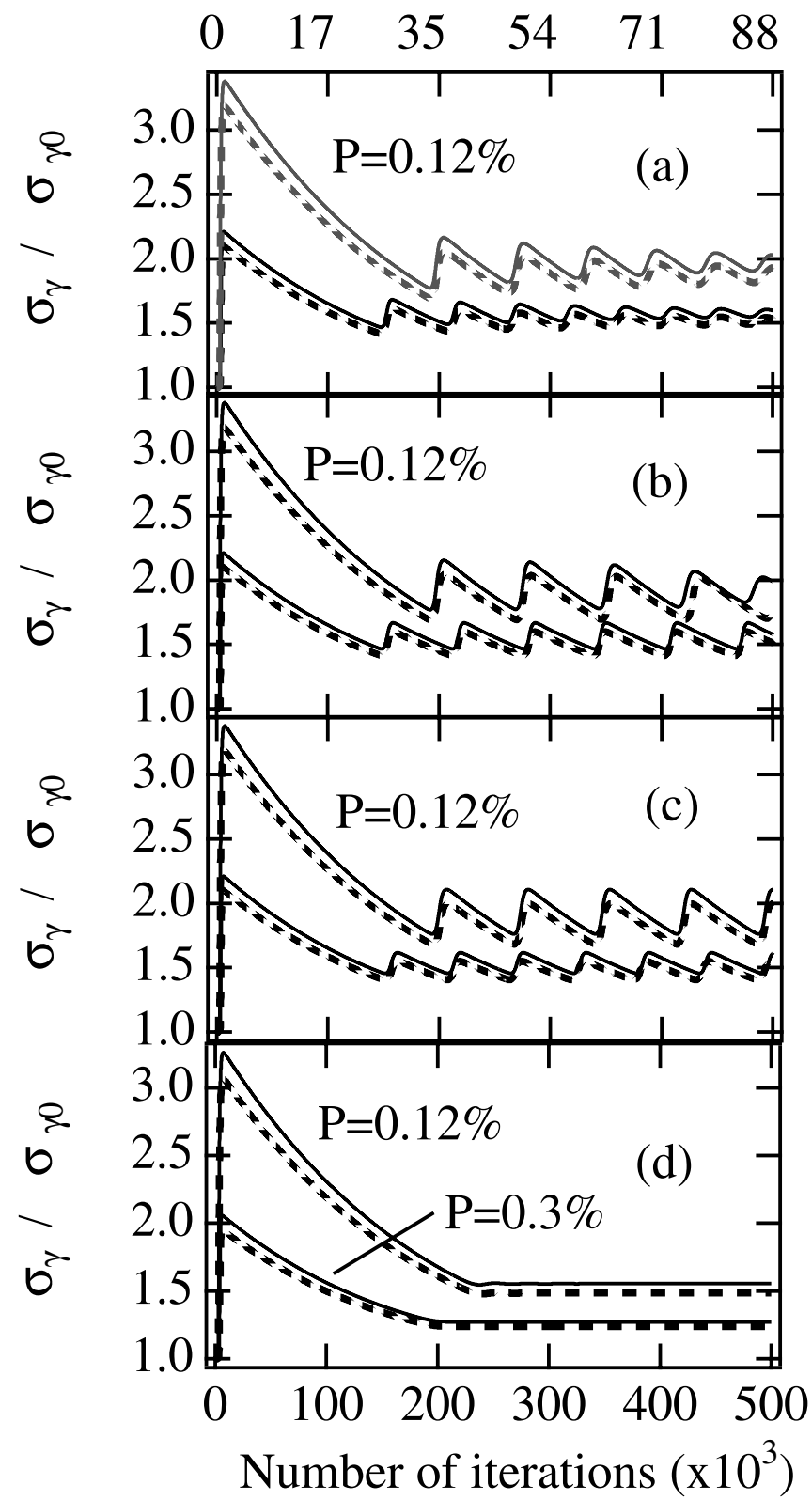

FIG. 9. Relative energy spread evolution with the LAS model for various detuning values: (a) $\delta=0 \mathrm{~Hz}$, (b) $\delta=2 \mathrm{~Hz}$, (c) $\delta=5 \mathrm{~Hz}$ (pulsed zone), (d) $\delta=40 \mathrm{~Hz}$, and two values of the losses: $P=0.12$ and $0.3 \%$. Continuous lines: achromatic point; dashed lines: chromatic point. An iteration corresponds to one revolution of the electron bunch in the storage ring. Simulations with etalas2v2, $I=10 \mathrm{~mA}, g_{0}=1 \%, N+N_{D}=87.5$. Those values correspond to experimental values.

LAS takes into account the natural bunch lengthening according to $\sigma_{l 0}=\alpha c \frac{\sigma_{\gamma 0}}{w_{S}}$, where $\sigma_{\gamma 0}$ is the experimental value measured at a current I. Simulations performed for different current values at $P=0.12 \%$ lead to a heated energy spread of $6.5 \times 10^{-4}$ (achromatic case) and $6.2 \times$ $10^{-4}$ (chromatic case) for $I=20 \mathrm{~mA}$, and of $1.2 \times 10^{-3}$ (achromatic case) and $1.1 \times 10^{-3}$ (chromatic case) for $I=$ $100 \mathrm{~mA}$, which is found in good agreement with the experimental results presented in Fig. 7. In order to improve the agreement of LAS simulations and experimental results, especially at high current, a more accurate description of electron beam longitudinal dynamic is foreseen, introducing the previously described vacuum chamber impedance influence on the electron beam dynamics. Analytical models have already been developed to introduce this impedance in the case of SLC [65] for potential well distortion, and Super-ACO [66] for microwave instability into the relative energy spread equation of evolution.

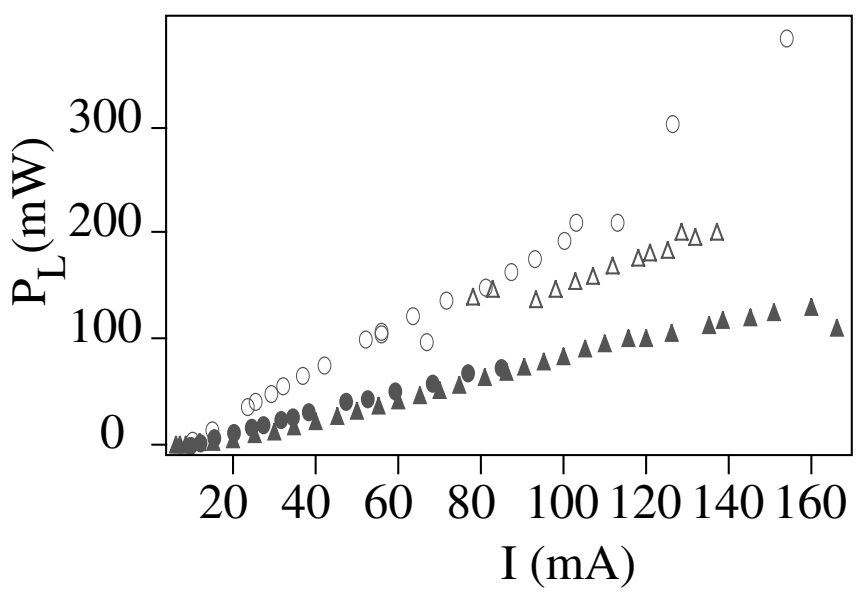

FIG. 10. FEL power versus current in the case of achromatic optics with $(\bigcirc) P=0.3 \%,(\bigcirc) P=0.12 \%$, and chromatic optics with $(\mathbf{\Delta}) P=0.3 \%,(\triangle) P=0.12 \%$.

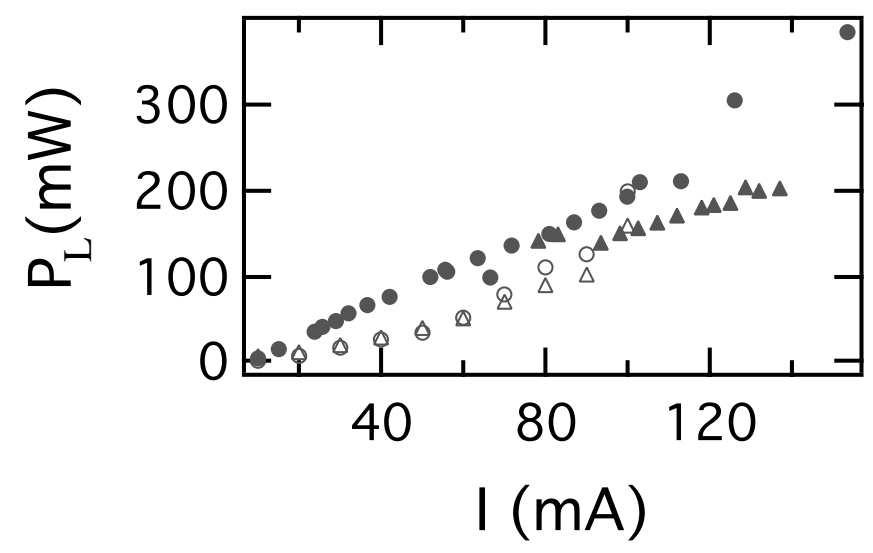

FIG. 11. FEL power calculated with LAS in achromatic and chromatic cases compared with the experimental results. (O) Experiment and $(\bigcirc)$ simulation in the achromatic case, $(\mathbf{\Delta})$ experiment and $(\triangle)$ simulation in the chromatic case. The parameters of the simulation are the same of the one used in Fig. 9, with $P=0.12 \%$. 


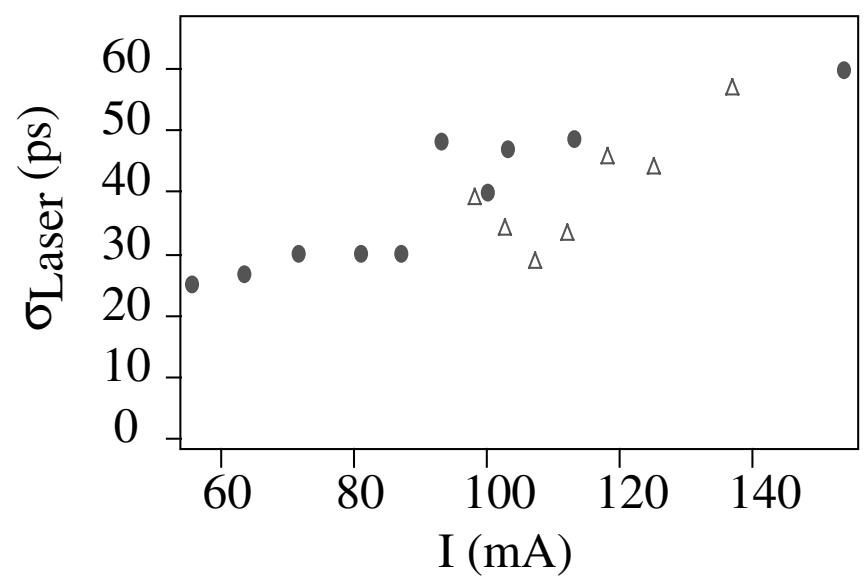

FIG. 12. FEL pulse duration in achromatic ( $)$ and chromatic $(\triangle)$ cases. The rms value of this distribution was calculated via the momenta method.

\section{ANALYSIS OF THE FEL POWER}

The output FEL power is directly related to the saturation process, and more precisely to the energy spread enhancement. In the case of SRFELs, the power is limited to a fraction of the synchrotron radiation power, because of the recirculation of the electron beam on many passes. It has been derived by Renieri and Vinokurov [67] as:

$$
P_{L}=\eta_{c} 8 \pi\left(N+N_{d}\right) f\left(\sigma_{\gamma e}^{2}-\sigma_{\gamma 0}^{2}\right) P_{S}
$$

with $\eta_{c}$ the extraction coefficient (ratio between mirror transmission and total losses). $P_{S}=6.04 \times 10^{-9} \frac{\gamma^{4} I}{\rho}$ is the synchrotron radiation power emitted at each pass, where $I$ is the current per bunch, $\rho$ the bending radius of the dipoles. The FEL power reflects the energy which has been transferred from the electron beam to the laser. The application of the Renieri limit is sometimes not straightforward, when the microwave instability takes place, and the initial energy spread to be considered has two limit cases: the natural energy spread and the energy spread at the given ring current. In fact, the FEL is competing in this case with the microwave instability [68] and can even prevent the growth of the energy spread and bunch length due to the wakefield, substituting its own induced heating to the one of the microwave instability. Here, in the UVSOR-II case, the potential well distortion regime is maintained for a wide range of current, making the application of the Renieri limit easier.

In Fig. 10 presenting the FEL performances for the two studied cases, the extracted power is smaller in the chromatic case than in the achromatic one, as a consequence of
Time (ms)

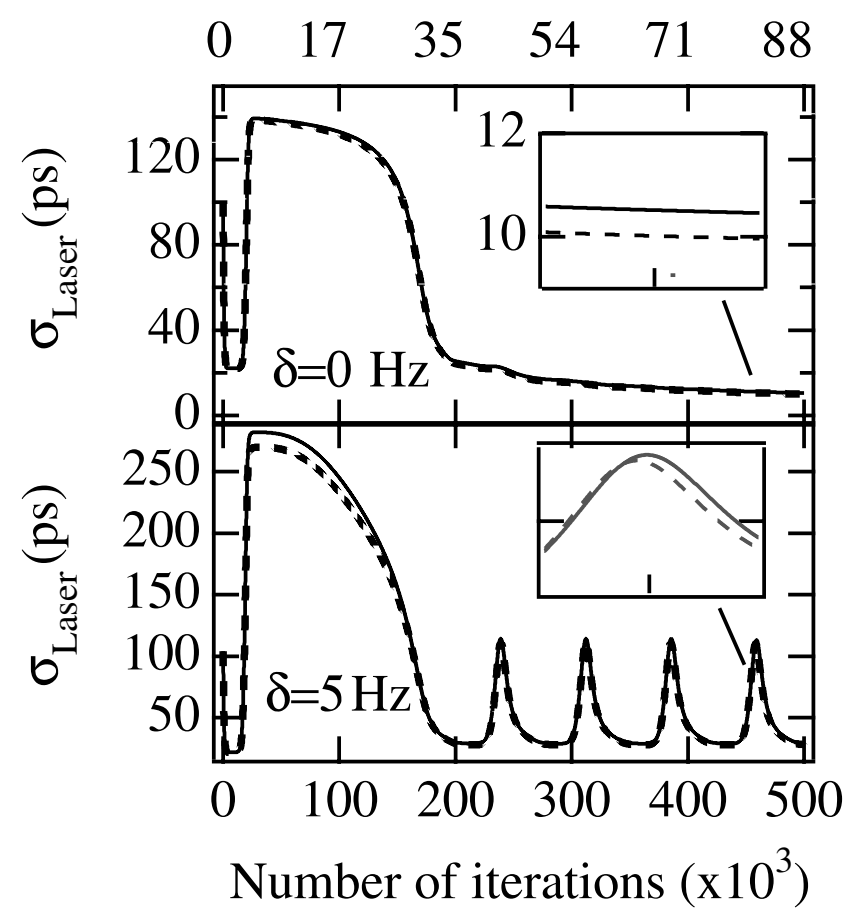

(a)
Time (ms)

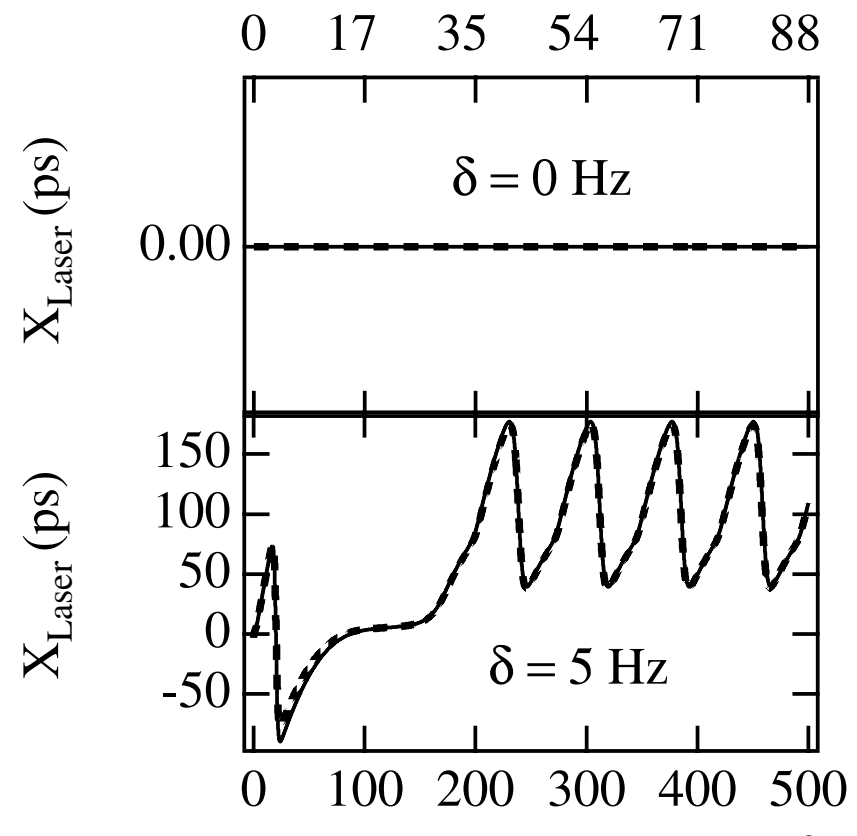

Number of iterations $\left(\times 10^{3}\right)$

(b)

FIG. 13. FEL features calculated with LAS in achromatic (continuous line) and chromatic (dotted line) cases for two detuning values: $\delta=0$ and $\delta=5 \mathrm{~Hz}$ (pulsed zone). Simulation performed for $I=10 \mathrm{~mA}$, and $P=0.12 \%$. (a) FEL pulse duration, (b) FEL center of mass position. 
the smaller energy spread enhancement required to achieve saturation, even though the gain is slightly higher. FEL power increases with current as expected from the Renieri limit. Calculations performed with Eq. (8) and using the Renieri limit are shown in Fig. 11, and found in qualitative good agreement with the experimental results. The dependence on the cavity losses also corresponds to the expectations, i.e. smaller output power for higher losses.

The analysis of the FEL power in the chromatic case also confirms the complexity of the saturation process, involving not only the energy spread and bunch length, but also the transverse sizes of the electron beam.

\section{FEL FEATURES}

Figure 12 illustrates experimental values of the laser pulse duration versus current in both operating points. The higher the current, the longer is the duration. Indeed, the bunch length, and so the possible interaction region with the laser pulse, increases with the current. A narrowing of the pulse duration is observed in the chromatic case at a given current.

Simulations performed with LAS enable one to visualize the evolution of this feature; as presented in Fig. 13(a), they confirm the experimental observed tendency. As developed previously, the introduction of a dispersion function leads to a reduced enhancement of the energy spread and as a consequence of the bunch length. Therefore, the longitudinal distribution of the gain along the laser pulse, given in LAS by $g(\tau)=g_{i} \exp \left(-\frac{(\tau+\delta)^{2}}{2 \sigma_{\tau}^{2}}\right)$, is narrower, and so is the laser intensity distribution delivered, from which is extracted the pulse duration. The pulse duration is then slightly reduced when using chromatic optics. Figure 13(b) shows the laser center of mass position. At perfect tuning, it corresponds to the electron bunch centroid since here, the slippage is negligible. For a small detuning $(5 \mathrm{~Hz})$, the laser is in a pulsed regime. In addition, the position in the chromatic case is shifted in time compared to the achromatic case. In fact, the transition time to reach the pulsed mode is longer in the chromatic case, explaining such a shift of the time evolution of the position. Finally, one can also notice that the frequency of the pulsed regime is different depending on the optics. In the $5 \mathrm{~Hz}$ simulated case of Fig. 13, the frequency is $77.2 \mathrm{~Hz}$ in the chromatic case against $76.9 \mathrm{~Hz}$ in the achromatic one. This can suggest an impact of the optics on the evolution of the dynamics versus detuning.

The use of chromatic optics, by changing the saturation process, also influences the final characteristics of the FEL output radiation.

\section{CONCLUSION}

This work confirms the evidence of a new saturation process, involving not only the electron beam longitudinal dynamics, but also the transverse one, via the heating of energy spread and the associated bunch lengthening, and an increase of the electron beam transverse sizes. The interplay between the FEL and the electron beam dynamics is even more complex than in the usual cases, and the FEL interaction can then be considered as a complex loop reacting on the global dynamics of the electron beam. It affects all the main electron beam characteristics. The saturation appears to occur for smaller energy spread enhancement when the transverse dynamics is involved. The understanding of such a complex interplay between the electron beam and a short laser pulse in the case of modern optics of synchrotron radiation sources in quest of the smallest emittance as possible is of interest not only for the storage ring FEL operation, but also for slicing [69] experiments, aiming at providing femtosecond synchrotron radiation by interaction of an ultrashort laser with the electron beam in an undulator, or for coherent harmonic generation experiments [70]. The presence of the distributed dispersion in the machine leads to a more complex interaction between electron and photon beams. Such a mechanism allows the electron beam features to be controlled, via the FEL heating, which can be adjusted in playing on the values of the gain with respect to the cavity losses, or on the detuning of the FEL. This present study provides further introduction of longitudinal to transverse coupling in the saturation process. More generally, it allows a better understanding of the interaction between the electron beam and a laser pulse, such as for slicing, harmonic generation, Compton scattering experiments [71]. Further accurate description with LAS is foreseen, as well as the introduction of the FEL induced heating in sophisticated storage ring codes such as TRACY [72].

\section{ACKNOWLEDGMENTS}

The authors would like to thank the technical staff of UVSOR-II facility: T. Murakami, K. Hayashi, and J. Yamazaki, as well as S. Bielawski and C. Szwaj who took part in the experiment, and C. Bruni for her helpful advice.

\section{APPENDIX}

The form of Haissinski equation [73], i.e. the static solution of the Fokker-Planck equation, depends on the wakefield $W_{w}$ given by the Fourier transform of the storage ring impedance [74]. It can be expressed as [75]: $\rho(\xi)=$ $A \exp \left(-\frac{\xi^{2}}{2}-S \int_{\xi}^{\infty} d \xi^{\prime} \int_{\xi^{\prime}}^{\infty} d \xi^{\prime \prime} \rho\left(\xi^{\prime \prime}\right) W_{w}\left(\xi^{\prime}-\xi^{\prime \prime}\right)\right.$, where $S$ is a constant depending on the nature of the wakefield. The beam distribution $\rho$ is a function of the normalized longitudinal position $\xi$, which is linked to the position $z$ of the electron with respect to the synchronous particle by: $\xi=\frac{w_{S}}{\alpha c \sigma_{\gamma 0}} z, c$ being the speed of light. The storage ring may be described by different impedance models in terms of LCR circuits. A good approximation is usually realized 
with a resistive-inductive impedance. In the case of a purely inductive case, the impedance is given by: $V=$ $-L \frac{d I}{d t}, I$ being the beam current distribution, and $L$ the ring inductance. The Haissinki equation becomes:

$$
\frac{d y}{d x}=-\frac{x y}{1+y},
$$

where $x=\frac{t}{\sigma_{l 0}}$, and $y=\frac{L I}{\left(V^{\prime} \sigma_{l 0}\right)^{2}}, V^{\prime}$ standing for the derivative of $V$ with respect to time, and the normalized charged can be defined as $\Gamma=\int_{-\infty}^{+\infty} y d x=\frac{L Q}{\left(V^{\prime} \sigma^{3}{ }^{1}\right)}$. The resolution of (A1) leads to a relation between $y$ and $x$, and therefore to the rms value $\sigma^{*}{ }_{l}$ of the $y(x)$ distribution. The third power fit of the $\Gamma\left(\sigma^{*}{ }_{l}\right)$ relation gave:

$$
\Gamma\left(\sigma_{l}^{*}\right)=8.44-22.1\left(\frac{\sigma_{l}^{*}}{\sigma_{l 0}}\right)+13.7\left(\frac{\sigma_{l}^{*} l}{\sigma_{l 0}}\right)^{2} .
$$

In the case of a finite induced energy spread, $\sigma_{l 0}$ can be replaced by $\varepsilon \sigma_{l 0}$, where $\varepsilon$ is the relative energy spread (energy spread divided by the natural energy spread), and Eq. (A2) becomes:

$$
\Gamma\left(\sigma_{l}\right)=\frac{L Q}{V^{\prime} \varepsilon^{3} \sigma_{l 0}^{3}}=6.48\left[\frac{1}{\varepsilon^{3}}\left(\frac{\sigma_{l}}{\sigma_{l 0}}\right)^{3}-1\right] .
$$

Then $\varepsilon$ can be written as:

$$
\varepsilon=\sqrt[3]{\left(\frac{\sigma_{l}}{\sigma_{l 0}}\right)^{3}-\frac{1}{6.48} \times \frac{L Q}{V^{\prime} \sigma_{l 0}{ }^{3}} .}
$$

The energy spread can then be deduced from Eq. (A4) once knowing the beam current and the bunch length. In the case of UVSOR, the inductance of $120-125 \mathrm{nH}$ is determined from the bunch lengthening data. The result of this energy spread measurement method is presented in Fig. 4 and reveals to be in good agreement with the other methods presented before.

[1] D. A. G. Deacon, L. R. Elias, J. M. J. Madey, G. L. Ramian, H. A. Schwettman, and T. I. Smith, Phys. Rev. Lett. 38, 892 (1977).

[2] V. Schlott, M. Dach, C. David, B. Kalantari, M. Pedrozzi, and A. Streun, in Proceedings of the European Particle Accelerator Conference, Lucerne, Switzerland, 2004, pp. 2526-2528.

[3] N. A. Papadogiannis, C. Kalpouzos, E. Goulielmakis, G. Nersisyan, D. Charalambidis, F. Augé, F. Weihe, and Ph. Balcou, Appl. Phys. B 73, 687 (2001).

[4] Ph. Zeitoun, G. Faivre, S. Sebban, T. Mocek, A. Hallou, M. Fajardo, D. Aubert, Ph. Balcou, F. Burgy, D. Douillet, S. Kazamias, G. de Lachèze-Murel, T. Lefrou, S. Le Pape, P. Mercère, H. Merdji, A. S. Morlens, J. P. Rousseau, and C. Valentin, Nature (London) 431, 426 (2004).

[5] G. De Ninno, E. Allaria, M. Marsi, and M. Trovo, in Proceedings of the Free Electron Laser Conference, Stanford, California, 2005 (to be published).
[6] K. J. Kim, Phys. Rev. Lett. 57, 1871 (1986).

[7] L. H. Yu, L. Di Mauro, A. Doyuran, W. S. Graves, E. D. Johnson, R. Heese, S. Krinsky, H. Loos, J. B. Murphy, G. Rakowsky, J. Rose, T. Shaftan, B. Sheehy, J. Skaritka, X. J. Wang, and Z. Wu, Phys. Rev. Lett. 91, 074801 (2003).

[8] X. J. Wang, H. Loos, J. B. Murphy, G. Rakowsky, J. Rose, B. Sheehy, Y. Shen, J. Skarita, Z. Wu, and L. H. Yu, in Proceedings of the Free Electron Laser Conference, Trieste, Italy, 2004, pp. 209-211.

[9] S. Schreiber, in Proceedings of the Free Electron Laser Conference, Stanford, California, 2005 [Report No. MOOB002 (to be published)].

[10] A. Nadji, in Proceedings of the European Particle Accelerator Conference, Vienna, Austria, 2000, pp. 1057-1059.

[11] L. Farvacque, J. L. Laclare, P. Nghiem, J. Payet, A. Ropert, H. Tanek and A. Tkatchenko, in Proceedings of the European Particle Accelerator Conference, London, UK, 1994, pp. 612-615.

[12] M. Katoh, K. Hayashi, T. Honda, Y. Hori, M. Hosaka, T. Kinoshita, S. Kouda, Y. Takashima, and J. Yamazaki, Nucl. Instrum. Methods Phys. Res., Sect. A 467-468, 68 (2001).

[13] N. A. Vinokurov and A. N. Skrinsky, INP77.59, Novossibirsk, 1977.

[14] P. Elleaume, J. Phys. (Paris), Colloq. 44, C1-353 (1983).

[15] A. Renieri, Nuovo Cimento B 53, 160 (1979).

[16] G. Dattoli and A. Renieri, Nuovo Cimento B 59, 1 (1980).

[17] M.E. Couprie and P. Elleaume, Nucl. Instrum. Methods Phys. Res., Sect. A 259, 77 (1987).

[18] V.N. Litvinenko, Y. Wu, B. Bentley, S. H. Park, M. Emamian, J. Faircloth, S. Goetz, N. Hower, J. M. Madey, J. Meyer, P. Morcombe, O. Oakeley, J. Patterson, R. Sachtschale, G. Swift, P. Wang, I. V. Pinayev, M. G. Fedotov, N. G. Gavrilov, V. M. Popik, V. N. Repkov, L. G. Isaeva, G. N. Kulipanov, G. Y. Kurkin, S.F. Mikhailov, A. N. Skrinsky, N. A. Vinokurov, P.D. Vobly, E. I. Zinin, A. H. Lumpkin, and B.X. Yang, in Proceedings of the SPIE Conference, San Jose, CA, 1997, pp. 188-199.

[19] M. Billardon, M.E. Couprie, D. Nutarelli, G. Flynn, P. Marin, R. Roux, and M. Sommer, in Proceedings of the European Particle Accelerator Conference, Stockholm, 1998, pp. 679-681.

[20] G. De Ninno, M. Trovo, M. Danailov, M. Marsi, and B. Diviacco, in Proceedings of the European Particle Accelerator Conference, Paris, France, 2002, pp. 799801.

[21] M. Hosaka, J. Yamazaki, T. Kinoshita, and H. Hama, in Proceedings of the Asian Particle Accelerator Conference, Tsukuba, Japan, 1998, p. 426.

[22] M. Billardon, D. Garzella, and M. E. Couprie, Phys. Rev. Lett. 69, 2368 (1992).

[23] V. Litvinenko, S. H. Park, I. V. Pinayev, and Y. Wu, Nucl. Instrum. Methods Phys. Res., Sect. A 475, 240 (2001).

[24] G. De Ninno, D. Nutarelli, D. Garzella, and M. E. Couprie, Phys. Rev. E 65, 056504 (2002).

[25] M.E. Couprie, M. Velghe, D. Jaroszynski, and M. Billardon, Nucl. Instrum. Methods Phys. Res., Sect. A 304, 58 (1991). 
[26] G. Dattoli, L. Mezi, and A. Renieri, Nucl. Instrum. Methods Phys. Res., Sect. A 393, 70 (1997).

[27] R. Bartolini, G. Dattoli, L. Mezi, A. Renieri, M. Migliorati, M.E. Couprie, R. Roux, and G. De Ninno, Phys. Rev. Lett. 87, 134801 (2001).

[28] G. Dattoli, L. Mezi, A. Renieri, M. Migliorati, M.E. Couprie, R. Roux, D. Naturelli, and M. Billardon, Phys. Rev. E 58, 6570 (1998).

[29] R. Bartolini, G. Dattoli , L. Giannessi, L. Mezi, C. Bruni, M.E. Couprie, D. Garzella, G. L. Orlandi, and M. Migliorati, Phys. Rev. E 69, 036501 (2004).

[30] G. L. Orlandi, C. Bruni, D. Garzella, M. E. Couprie, G. De Ninno, R. Bartolini, L. Gianessi, and G. Dattloli, Nucl. Instrum. Methods Phys. Res., Sect. A (D-05-00129R1, to be published).

[31] C. Bruni, G. De Ninno, D. Garzella, A. Nadji, C. Thomas, M.E. Couprie, R. Bartolini, and L. Giannessi, Nucl. Instrum. Methods Phys. Res., Sect. A 483, 167 (2002).

[32] M. Billardon, P. Elleaume, J. M. Ortega, C. Bazin, M. Bergher, M. Velghe, Y. Petroff, D. A. G. Deacon, K. E. Robinson, and J. M. J. Madey, Phys. Rev. Lett. 51, 1652 (1983).

[33] G. N. Kulipanov et al., Nucl. Instrum. Methods Phys. Res., Sect. A 296, 1 (1990).

[34] K. Yamada et al., Nucl. Instrum. Methods Phys. Res., Sect. A 318, IN1 (1992).

[35] D. Nolle, D. Garzella, A. Geisler, L. Gianessi, M. Hirsh, H. Quick, M. Ridder, T. Schmidt, and K. Wille, Nucl. Instrum. Methods Phys. Res., Sect. A 445, 128 (2000).

[36] T. Yamasaki, K. Yamada, S. Sugiyama, H. Ohgaki, N. Sei, T. Mikado, T. Noguchi, M. Chiwaki, and R. Suzuki, Nucl. Instrum. Methods Phys. Res., Sect. A 331, 27 (1993).

[37] V. Litvinenko, Nucl. Instrum. Methods Phys. Res., Sect. A 407, 8 (1998).

[38] M.E. Couprie, C. Bazin, M. Bergher, M. Billardon, P. Elleaume, J. M. Ortéga, Y. Petroff, R. Prazeres, and M. Velghe, Nucl. Instrum. Methods Phys. Res., Sect. A 282, 455 (1989).

[39] S. Takano and H. Hama, Nucl. Instrum. Methods Phys. Res., Sect. A 331, 20 (1993).

[40] R.P. Walker, J.A. Clarke, M.E. Couprie, G. Dattoli, M. Eriksson, D. Garzella, L. Giannessi, M. Marsi, D. Nlle, D. Nutarelli, M. W. Poole, H. Quick, E. Renault, R. Roux, M. Trovò, S. Werin, and K. Willeand, Nucl. Instrum. Methods Phys. Res., Sect. A 475, 20 (2001).

[41] M. Sands, The Physics of Electron Storage Rings. An Introduction (University of California, Santa Cruz, California, 1971), Chap. 5, pp. 370-380.

[42] J. A. Clarke, H. L. Owen, M. W. Poole, S. L. Smith, and V.P. Suller, in Proceedings of the European Particle Accelerator Conference, Stockholm, 1998, pp. 253-255.

[43] Y. Kamiya and M. Kihara, KEK Report No. 83-16, 1983 (unpublished).

[44] A. Nadji, see Ref. [10].

[45] Y. Papaphilippou, A. Ropert, P. Elleaume, and L. Farvacque, in Proceedings of the Particle Accelerator Conference, Knoxville, Tennessee, 2005, pp. 2047-2049.

[46] M. Katoh, K. Hayashi, Y. Hori, M. Hosaka, T. Kinoshita, A. Mochihashi, Y. Takashima, and J. Yamasaki, in
Proceedings of the Asian Particle Accelerator Conference, Beijing, 2001, pp. 296-298.

[47] M.P. Level, P. Brunelle, R. Chaput, J. M. Filhol, C. Herbeaux, A. Loulergue, O. Marcouillé, J. L. Marlats, A. Nadji, and M. A. Tordeux, in Proceedings of the European Particle Accelerator Conference, Paris, France, 2002, pp. 212-214.

[48] A. Wolski, in Proceedings of the European Particle Accelerator Conference, Vienna, Austria, 2000, pp. 1995-1998.

[49] H. Ohkuma, S. Date, M. Masaki, T. Nakamura, T. Oshima, K. Soutome, S. Takano, K. Tamura, M. Takao, H. Tanaka, and N. Kumaiga, in Proceedings of the Particle Accelerator Conference, Portland, Oregon, 2003, pp. 881-883.

[50] A. Jackson, in Proceedings of the Particle Accelerator Conference, Knoxville, Tennessee, 2005, pp. 102-106.

[51] J. Bordas, J. Campmany, D. Einfeld, S. Ferrer, M. Munoz, F. Pérez, and M. Pont, in Proceedings of the European Particle Accelerator Conference, Lucerne, Switzerland, 2004, pp. 2326-2328.

[52] K. Hirata, CERN 88-04, 1988.

[53] K. Kubo, H. Hayano, S. Kamada, M. Kuriki, S. Kuroda, T. Naito, T. Okugi, N. Terunuma, N. Toge, J. Urakawa, and M. Takano, in Proceedings of the European Particle Accelerator Conference, Vienna, Austria, 2000, pp. 483485.

[54] H. Wiedemann, Particle Accelerator Physics (Springer, New York, 1998), Vol. II.

[55] B. Zotter, CERN SPS/81-14 (DI), 1981.

[56] C. Boussard, Internal Report CERN 75-2, 1975 (unpublished).

[57] A. Mochihashi, M. Katoh, M. Hosaka, K. Hayashi, J. Yamasaki, and Y. Takashima, UVSOR Activity Report 2003, 2004, p. 35.

[58] J. M. J. Madey, J. Appl. Phys. 42, 1906 (1971).

[59] W. B. Colson, Laser Handbook: Free Electron Lasers (North-Holland, Amsterdam, 1990), Vol. 6, Chap. 5.

[60] W. B. Colson and P. Elleaume, Appl. Phys. B 29, 101 (1982).

[61] D. Nutarelli, Ph.D. thesis, University of Paris XI, Orsay, 2000, Chap. 1, p. 46-50.

[62] C. Bruni, Ph.D. thesis, University of Paris XI, Orsay, 2004.

[63] G. De Ninno, D. Fanelli, C. Bruni, and M. E. Couprie, Eur. Phys. J. D 22, 269 (2003).

[64] S. Bielawski, C. Swaj, C. Bruni, D. Garzella, G. L. Orlandi, and M.E. Couprie, Phys. Rev. Lett. 95, 034801 (2005).

[65] K. L.F. Bane and K. Oide, in Proceedings of the Particle Aceelerator Conference, Washington D.C., 1993, pp. 3339-3341.

[66] R. Roux and M. Billardon, Nuovo Cimento A 112, 513 (1999).

[67] N.A. Vinokurov et al., in Proceedings of the SRI-88 Conference, Tsukuba (1988).

[68] G. L. Orlandi, C. Bruni, D. Garzella, M.E. Couprie, C. Thomas, R. Bartolini, C. Rippon, and G. Dattoli, Phys. Rev. ST Accel. Beams 7, 060701 (2004).

[69] S. Khan and H. A. Dürr, in Proceedings of the European Particle Accelerator Conference, Paris, France, 2002, pp. 700-703. 
[70] J. Y. Liu, C.Z. Diao, D. H. He, Q. K. Jia, G. Li, H. L. Xu, P. F. Zhang, and S. C. Zhang, in Proceedings of the Particle Accelerator Conference, Stanford, California, 2003, pp. $968-970$.

[71] M. A. Beno, C. Kurtz, A. Munkholm, U. Rütt, M. Engbreston, G. Jennings, J. Linton, G. S. Knapp, and P. A. Montano, Nucl. Instrum. Methods Phys. Res., Sect. A 467, 694 (2001).

[72] H. Nishimura, in Proceedings of the International
Workshop on Controls for Small and Medium-Scale Accelerators, KEK Tsukuba, 1996.

[73] J. Haïssinski, Nuovo Cimento Soc. Ital. Fis. B 18, 72 (1973).

[74] A. Hofmann, in Proceedings of the CERN Accelerator School (CERN, Geneva, 1995), Vol. 95-06, Chap. 14.

[75] C. Thomas, Ph.D. thesis, Université Paris XI, Orsay, 2003, Chap. 3, p. 38 . 